\title{
Improving the Lifetime of Mechanical System Such as Hinge Kit System Subjected to Repeated Impact Stress
}

\author{
Seongwoo Woo ${ }^{1 *}$, Dereje Engida Woldemichael ${ }^{1}$, Samson Mekbib Atnaw ${ }^{1}$, Muluneh Mekonnen Tulu ${ }^{1}$ and \\ Dennis L 0'Neal ${ }^{2}$
}

${ }^{1}$ Faculty of Mechanical Engineering, Addis Ababa Science \& Technology University, Addis Ababa, Ethiopia

${ }^{2}$ Dean of Engineering and Computer Science, Baylor University, USA

*Corresponding author: Seongwoo Woo, Faculty of Mechanical Engineering,

Addis Ababa Science \& Technology University, Addis Ababa, Ethiopia.

Received Date: November 25, 2020

Published Date: December 08, 2020

\begin{abstract}
To enhance the lifetime of mechanical system, parametric Accelerated Life Testing (ALT) as new systematic reliability method suggests evaluating the design of mechanical systems subjected to repeated stresses, based on failure mechanism and design. This newly developed parametric ALT helps an engineer uncover the missing design parameters (or design flaws) of the mechanical system having an effect on reliability during the product developing process. As a result, companies can circumvent product recalls due to the design flaws from the marketplace. As an experiment instance, the redesign of hinge kit system (HKS) in a domestic refrigerator was investigated.
\end{abstract}

Keywords: Reliability design; HKS; Fracture; Parametric ALT; Design flaws

\section{Introduction}

The mechanical products such as automobile, airplane, and refrigerator manage power to achieve a job that requires forces and movement, which produce mechanical advantages by adapting product mechanisms. For example, using the vapor-compression refrigeration system, a refrigerator supplies chilled air from the evaporator to the refrigerator and freezer section. Customers want a refrigerator that have high performance and reliability for multi-functions. Accordingly, a HKS structured as a spring-damper therefore was designed as a consumer want to gently open and close a refrigerator door with minimal effort. If there is design fault in the system when the loads are exerted, HKS may unexpectedly fail in its anticipated lifetime. After recognizing the product failure by parametric ALT, an engineer might most favorably design. This study proposes a parametric ALT as systematic reliability method that can be applicable to mechanical systems. It composes: (1) a parametric ALT plan, (2) a load analysis, (3) a customized ALTs with the design alterations, and (4) an assessment of whether the product design(s) achieves the objective BX lifetime. As an experiment instance, we will discuss as following: the redesign of HKS in a refrigerator.

\section{Parametric ALT in Mechanical System}

If there is a wrong design in the product where the (dynamic) loading are exerted, it might suddenly be unsuccessful during its lifetime. After recognizing the market failure by parametric ALTs, an engineer will design the parts such as material pattern to overcome its own loads.

$$
A F=\left(\frac{S_{1}}{S_{0}}\right)^{n}\left[\frac{E_{a}}{k}\left(\frac{1}{T_{0}}-\frac{1}{T_{1}}\right)\right]=\left(\frac{e_{1}}{e_{0}}\right)^{\lambda}\left[\frac{E_{a}}{k}\left(\frac{1}{T_{0}}-\frac{1}{T_{1}}\right)\right] \text { (1) }
$$

To get the required mission cycle of an ALT, sample size formulation with the AF might be defined as [1]: 


$$
n \geq(r+1) \cdot \frac{1}{x} \cdot\left(\frac{L_{B X}^{*}}{A F \cdot h_{a}}\right)^{\beta}+r(2)
$$

\section{Case Study: Reliability Design of a HKS Subjected to Repeated Stresses}

As a customer utilizes the refrigerator, one would close its door comfortably. For this purpose, the HKS is newly designed (Figure 1).

From the marketplace, HKS components in a domestic refrigerator were failing due to design problems. Formed on the customer working states, HKS were subjected to dissimilar loading during the operations of the refrigerator door (Figure 2).

The moment balance at the HKS might be defined as

$$
\begin{aligned}
& M_{0}=W_{d o o r} \times b(3 \mathrm{a}) \\
& \text { (3a) }=T_{0}=F_{0} \times R(3 \mathrm{~b})
\end{aligned}
$$

If accelerated loads on the refrigerator door is attached, the moment balance at the HKS might be redefined as

$$
\sum M=M_{1}=M_{0}+M_{A}=W_{\text {door }} \times b+W_{A} \times a=T_{1}=F_{1} \times R
$$

$$
(4 \mathrm{a})=T_{1}=F_{1} \times R(4 \mathrm{~b})
$$

Under the same working conditions, the life-stress model might be expressed as:

$$
T F=A(S)^{-n}=A T^{-\lambda}=A(F \times R)^{-\lambda}=B(F)^{-\lambda}
$$

We recognized that system lifetime relies on exerted impact load. Consequently, the acceleration factor (AF) in Equation (1) might be defined as

$$
A F=\left(\frac{S_{1}}{S_{0}}\right)^{n}=\left(\frac{T_{1}}{T_{0}}\right)^{\lambda}=\left(\frac{F_{1} \times R}{F_{0} \times R}\right)^{\lambda}=\left(\frac{F_{1}}{F_{0}}\right)^{\lambda}
$$

If the lifetime target of the newly designed HKS was put to be B1 life 10 years, the number of needed test cycles can be acquired for assigned sample pieces in Equation (2). In ALTs the problematic designs of HKS in the product developing process might be recognized to fulfill the lifetime objective.

(a)
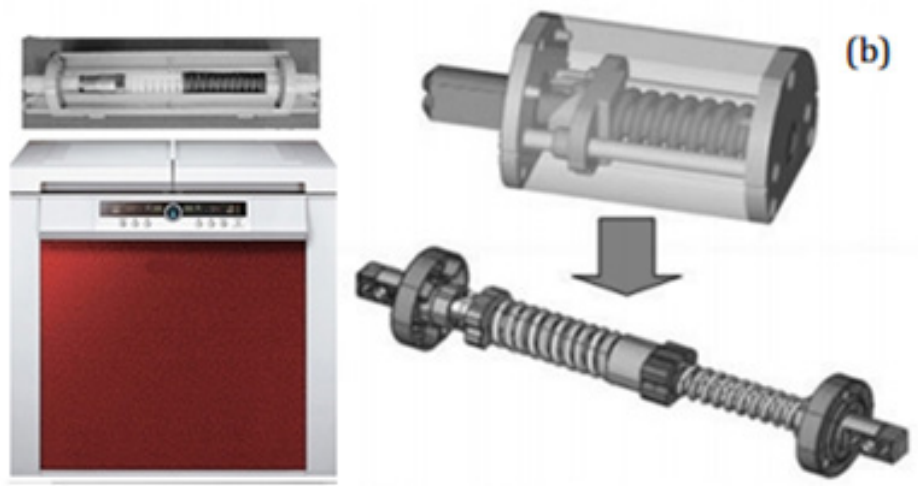

Figure 1: (a) Domestic refrigerator and (b) HKS.

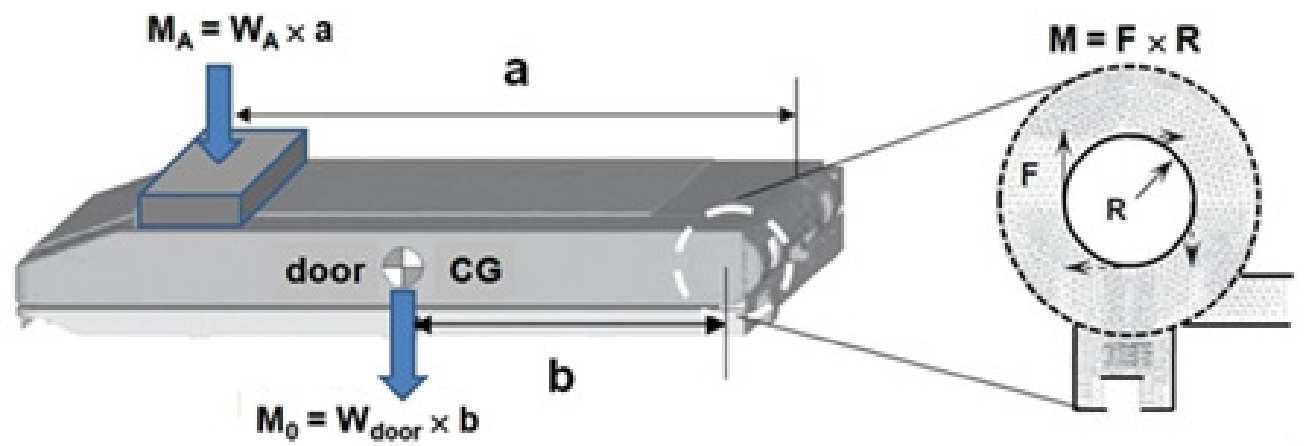

Figure 2: Design ideas of HKS.

\section{Results and Conclusions}

In the first ALT, the housing of the HKS failed at 3,000 cycles. Comparing the fractured product from the marketplace and that from the first ALT, we knew that the appearance and placement of the failure in the $1^{\text {st }}$ ALT were identical to those obtained from the marketplace (Figure 3). 

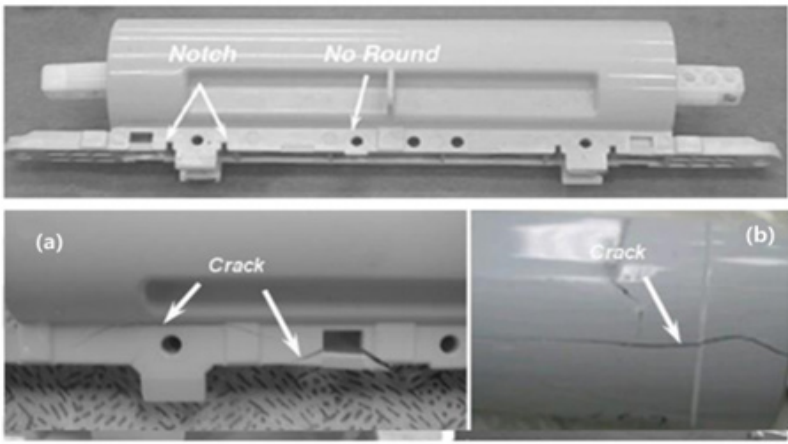

(a) Failed products from the marketplace (b) Fracture after $1^{\text {st }}$ ALT

Figure 3: Failed products from the marketplace and 1st ALT.

The fracture of the HKS in both the market products and $1^{\text {st }}$ ALT test samples happened at its housing. We knew that the design flaws of the HKS came from open structure in its housing there is no supporting ribs. To attain sufficient mechanical strength against repeated loading, the problematic hinge kit housing was modified as inside supporting ribs was attached (Figure 4).
In the second ALT, the torsional shaft in HKS failed at 12,000 cycles. We knew that the root cause of cracked torsional shaft for the $2^{\text {nd }}$ ALTs originated from its insufficient rounding due to repeated stress. As an action plans, to have sufficient material strength for repetitive impact loads, its torsional shaft was reshaped to give it more rounding from $\mathrm{R} 0.5 \mathrm{~mm}$ to $\mathrm{R} 2.0 \mathrm{~mm}$ (Figure 5).

Figure 4: Redesigned HKS housing structure.
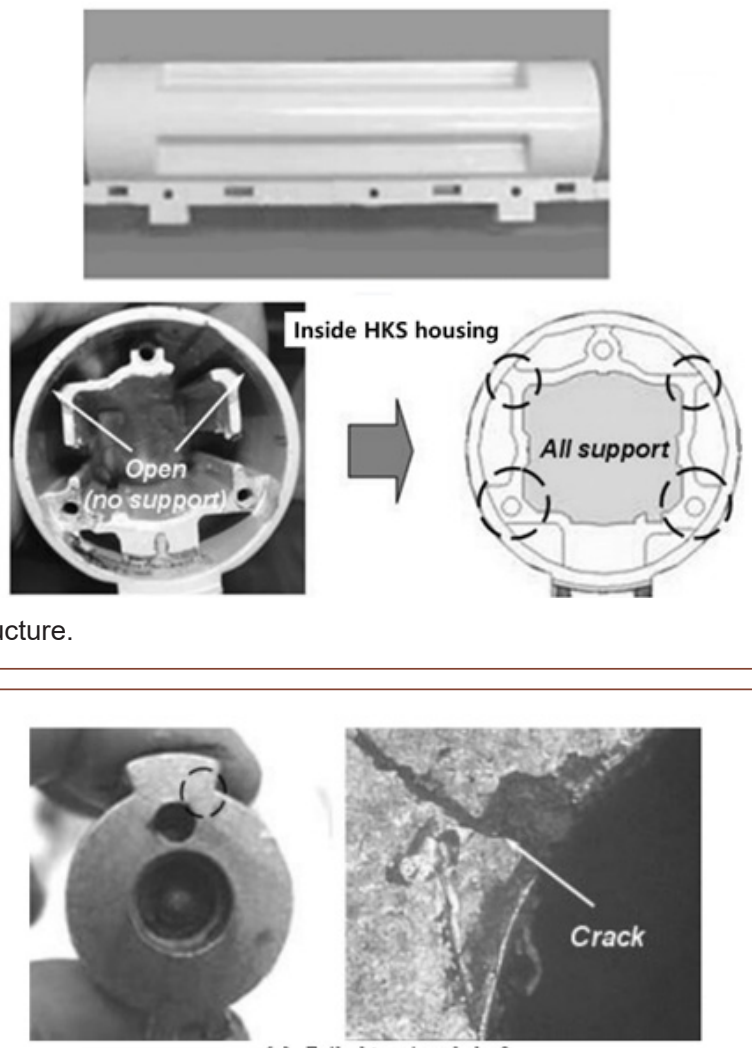

(a) Failed torsional shaft

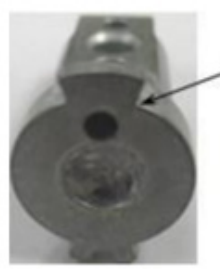

R0.5

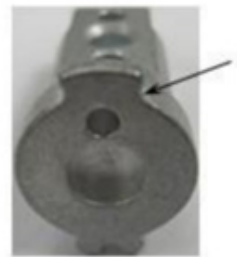

R2.0

Figure 5: Redesigned torsional shaft.

(b) Modified torsional shaft 
With the design alterations, the HKS in domestic refrigerator could properly work to satisfy the reliability need during its lifetime because there were no issues until 24,000 mission cycles. Over the course of three ALTs, the lifetime of samples were assured to be B1 life 10.0 years with an accumulated failure rate of $1 \%$.

\section{Acknowledgement}

None.

\section{Conflict of Interest}

No conflict of interest.

\section{References}

1. Woo S, Pecht M, O’Neal DL (2020) Reliability design and case study of the domestic compressor subjected to repetitive internal stresses. Reliability Engineering and System Safety 193: 106604. 